\title{
Dissoziation bei Mensch und Maus
}

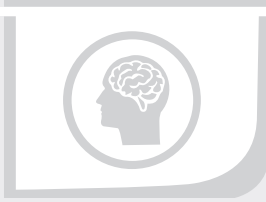

lisiert, dass man von der Fahrt gar nichts mehr weiß und sie scheinbar „wie auf Autopilot“ erfolgt ist, hat fast jeder schon einmal erlebt. Oder dass man mit jemandem spricht und plötzlich realisiert, dass man in Gedanken woanders war und für eine Weile gar nicht zugehört hat. Das Gefühl, die Welt sei irgendwie nicht wirklich, oder wie ein Film, kommt ebenfalls vor, wenngleich nicht so häufig. Das Gefühl, neben sich zu stehen und sich gleichsam selber zu betrachten (Autoskopie), ist auch eher selten. Zustände, die mit Worten wie „Trance“ oder „Besessenheit“ benannt werden, werden allerdings unter „Dissoziation“ ebenso verbucht wie Nahtoderfahrungen, die epileptische Aura vor einem Anfall, sogenannte „hysterische“ Lähmungen (heute als dissoziative Bewegungsstörungen bezeichnet) und andere „Syndrome“, die unter „Konversion“ bekannt sind, sowie das Ganser-Syndrom („wieviel Finger hat eine Hand?“ - „6“; „wieviel ist $4 \times 4$ ?“ - „15“; man spricht auch von Vorbeireden) und die multiple Persönlichkeit, sofern es diese tatsächlich gibt.

Im Hinblick auf den Ausprägungsgrad nimmt man ein Kontinuum von „geringgradig“ bis „stark“ an. Die beschriebenen, leichten dissoziativen Zustände hat nahe-

gebnisse eröffnen einen völlig neuen Horizont des Verständnisses von Dissoziation und katapultieren unser Nachdenken über Dissoziation vom vorletzten ins 21 . Jahrhundert.

Unter Dissoziation versteht man das Auseinanderfallen von psychischen Funktionen, die normalerweise zusammenhängen. Dabei können die verschiedensten geistigen Leistungen und Funktionen wie Wahrnehmung, Körperempfindungen (etwa Schmerz und Hunger), Bewusstsein, Gedächtnis, Identität und Motorik betroffen sein [29]. Dass man mit dem Auto zur Arbeit fährt, ankommt, und plötzlich rea-
- Tab. 1 Abkürzend wiedergegebene Beispiele von Dissoziation aus einem bekannten Fragebogen zu Dissoziationserfahrungen (Dissociative Experience Scale [4]).

zu jeder Mensch schon erlebt, schwere dissoziative Zustände hingegen kommen bei 2-4\% der Allgemeinbevölkerung, bei 15\% der ambulant und $30 \%$ der stationär behandelten psychiatrischen Patienten vor [28]. Die Häufigkeit der Symptome nimmt mit steigendem Alter ab. Dissoziative Bewegungsstörungen - psychogener Tremor, psychogene Dystonie, Myoklonie, Gangstörung, Ataxie - machen 2,6-25\% der Bewegungsstörungen in neurologischen $A b$ teilungen aus [15, 25].

Die Unschärfe des Begriffs der „Dissoziation“, die damit verbundene extreme Heterogenität der Sachverhalte, die darunter subsumiert werden ( $\triangleright$ Tab. 1) und die daraus zwangsläufig resultierenden sehr heterogenen empirischen Befunde haben in der Praxis zu viel Unsicherheit und nur wenigen wirklichen Fortschritten geführt. Dies könnte sich mit einer im Fachblatt Nature erschienen Arbeit zur Dissoziation in einem Mausmodel aus der Arbeitsgruppe um den bekannten Psychiater und Bioingenieur Karl Deisseroth von der Stanford University ändern, zumal am Ende der Arbeit die Erkenntnisse aus dem Labor auf einen Fall von Dissoziation beim Menschen übertragen wurden [10].

\begin{tabular}{|l|l|}
\hline Item-Nr. & Entscheiden Sie bitte, in wie viel Prozent Ihrer Zeit Sie diese Erfahrung machen \\
\hline 1 & $\begin{array}{l}\text {.. beim Autofahren merken, dass Sie nicht mehr wissen, was während der Fahrt } \\
\text { passierte. }\end{array}$ \\
\hline 3 & $\ldots$ sich plötzlich an einem Ort befinden, ohne zu wissen, wie Sie dahin kamen. \\
\hline 7 & $\ldots$ Gefühl, neben sich zu stehen oder sich selbst bei etwas zuzuschauen. \\
\hline 20 & $\ldots$ Menschen, Gegenstände und die Welt um sich herum nicht als wirklich empfinden. \\
\hline 24 & $\ldots$ in die Luft starren, ohne an etwas zu denken oder zu bemerken, wie die Zeit vergeht. \\
\hline 27 & $\begin{array}{l}\text {... hören Sie manchmal Stimmen im Kopf, die Ihnen Dinge sagen, die Sie tun sollen } \\
\text { oder kommentieren, was Sie gerade tun. }\end{array}$ \\
\hline 28 & \begin{tabular}{l} 
Gegenstähl, als ob Sie die Welt durch einen Nebel wahrnehmen, dass Menschen und \\
\hline
\end{tabular}
\end{tabular}


Karl Deisseroth machte sich als Methodiker einen Namen [31] und gilt als einer der Begründer der Optogenetik. Bei dieser Methode macht man sich lichtabhängige lonenkanäle, die in den Zapfen und Stäbchen der Augen auf Licht reagieren, zur neurobiologischen Grundlagenforschung zunutze. Bei diesen lonenkanälen handelt es sich um Eiweißkörper, die Channelrhodopsine genannt werden und die innerhalb von Millisekunden Aktionspotenziale bei Lichteinwirkung generieren können. Man kann nun die für diese Proteine kodierende genetische Information in Nervenzellen einschleusen, was dazu führt, dass diese Zellen gezielt dann durch Licht einer bestimmten Wellenlänge (Farbe) elektrisch aktiviert (depolarisiert) werden [10]. Auch eine Deaktivierung durch Licht anderer Wellenlänge kann mit entsprechenden hyperpolarisierenden, lichtabhängigen Proteinen herbeigeführt werden ${ }^{1}$ ( $>$ Abb. 5).

Wie untersucht man Dissoziation bei einer Maus? Die Phänomene scheinen einen menschlichen Geist vorauszusetzen, zu-

1 Die Methode wird von sehr vielen Arbeitsgruppen weltweit in der neurobiologischen Grundlagenforschung als auch zur Erforschung neurologischer und psychiatrischer Erkrankungen verwendet und macht Herrn Deisseroth nobelpreisverdächtig.

\section{KETAMIN}

Ketamin ist ein bei Mensch und Tier eingesetztes Medikament zur Anästhesie und Analgesie und wirkt in geringeren Dosen dissoziativ. Die weiße kristalline Substanz wurde im Jahr 1962 erstmals synthetisiert, weil man einen Ersatz für das bereits 6 Jahre zuvor entwickelte anästhetisch und analgetisch wirkende Phencyclidin (Phenylcyclohexylpiperidin, kurz PCP) suchte. Dieser Stoff wirkte ebenfalls dissoziativ, zeigte jedoch ein starkes Suchtpotenzial sowie neurotoxische Eigenschaften (mittlerweile auch beim Menschen mittels MRT nachgewiesen $[17,36])$, weswegen seine Vermarktung (im Jahr 1963) 4 Jahre nach der Einführung in der deutschen Humanmedizin wieder eingestellt wurde. In den USA wird PCP noch heute in der Tiermedizin eingesetzt und dort - als Angel Dust - mehr als mal die bei ihrer Beschreibung verwendeten theoretischen Vorannahmen (Ich-Bewusstsein, Konflikte, unbewusste Ängste, Abwehrmechanismen, Konversion) den von Sigmund Freud so bezeichneten „Psychischen Apparat“ implizieren. Deisseroth und Mitarbeiter machten sich zunächst die Tatsache zunutze, dass dissoziative Zustände medikamentös induziert werden können und verabreichten Mäusen Ketamin (Kasten: Ketamin) in subanästhetischen Dosen $(50 \mathrm{mg} / \mathrm{kg}$ ) intraperitoneal. Mit Hilfe der Methode des Calcium-Imaging ${ }^{2}$ wurde dann an einem Mauspräparat in vivo die neuronale Aktivität über einen weiten Bereich des freipräparierten Kortex

2 Bei dieser mittlerweile sehr häufig angewandten Methode wird Calmodulin, ein Kalzium bindendes Protein, über ein Peptid und weitere Verbindungsglieder („Linker“) mit einem Chromophor verbunden.

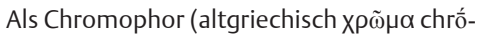
ma „Farbe“, popós phorós „tragend“) wird die gesamte farbgebende Atom- oder lonengruppierung einer chemischen Verbindung bezeichnet. Die Farbigkeit kann durch Lichtabsorption, Lichtreflexion, Lichtstreuung oder Lichtbrechung erfolgen. Durch Variationen in der Chemie wurden besonders sensible Stoffe gefunden, die mit Licht verschiedener Wellenlängen arbeiten und so beispielsweise beim visuellen System in Abhängigkeit von der Orientierung eines Streifenmusters verschiedenfarbig leuchten [7].

gemessen ( $\triangleright$ Abb. 3). Nach Gabe von Ketamin zeigte sich eine Oszillation von $1-3 \mathrm{~Hz}$ im retrosplenialen Kortex der Maus (Kasten: Retrosplenialer Kortex). Die synchrone rhythmische neuronale Aktivität begann etwa 2 Minuten nach der Ketamininjektion und dauert etwa 45 Minuten an.

Vor diesem Hintergrund ist von Bedeutung, dass bei den Mäusen unter Ketamin auch gefunden wurde, dass die Korrelationen zwischen der Aktivität des retrosplenialen Kortex und anderen (in der Präparation sichtbaren) dorsalen kortikalen Arealen abnahm. Weil Ketamin eine ganze Reihe von Wirkungen und Wirkungsmechanismen aufweist (Kasten: Ketamin), untersuchten die Autoren mit gleicher Methodik andere Substanzen, deren Effekte sich denen von Ketamin überlappen: NMDA-Rezeptorantagonisten (Phencyclidin, Dizocilpin und Memantin), ein Halluzinogen (LSD), Anästhetika (Dexmedetomidin und Propofol), ein Sedativum (Diazepam) und ein Analgetikum (Buprenorphin). Nur die beiden ebenfalls dissoziative Zustände herbeiführenden Substanzen Phencyclidin (PCP) und Dizocilpin (bekannt unter der Bezeichnung MK-801) verursachten die retrosplenialen Oszillationen wie Ketamin, nicht hingegen der - keine dissoziativen Zustände hervorrufende - NMDA-Rezeptorantago-

anderswo illegal verwendet [18]. Die Substanz führt zu psychotischen Symptomen (sowohl Positiv-Symptome wie Halluzinationen, Ich-Störungen, gesteigerter Antrieb sowie formale und inhaltliche Denkstörungen als auch Negativ-Symptome wie Apathie, Anhedonie, Affektverarmung und Antriebsminderung). Weil PCP zu Aggressivität bis hin zu Tobsuchtsanfällen mit Eigen- und Fremdgefährdung bei gleichzeitiger Schmerzunempfindlichkeit und nachfolgenden Gedächtnislücken führen kann, wird sein Konsum auch mit Amokläufen und Massakern in Verbindung gebracht.

Im Jahr 1964 führte der klinische Pharmakologe Edward Felix Domino einen Selbstversuch mit Ketamin durch und bezeichnete seine Wirkungsweise als dissoziatives Anästhetikum, womit in der Anästhesie gemeint ist, dass einerseits Schlaf (Bewusstseinsverlust) und
Schmerzfreiheit herbeigeführt werden, andererseits die Schutzreflexe intakt bleiben und damit keine Aspirationsgefahr besteht. Zudem besteht auch eine geringere Gefahr eines Atemstillstandes. Ein Jahr später begann man dann mit der Erprobung von Ketamin im schon seit 10 Jahren laufenden Vietnamkrieg zur Behandlung von verwundeten Soldaten. Sein häufiger erfolgreicher Einsatz in der Schmerzbekämpfung bei gleichzeitiger Erhaltung der Schutzreflexe - also seine zur Dissoziation führende Wirkung - führte im Jahr 1970 zu seiner Zulassung in den USA. Mit dem Ende des Vietnamkriegs im Jahr 1975 verbreitete sich Ketamin in den USA als Straßendroge - kein Wunder: Seit 1973 waren über eine halbe Million US-Soldaten mit entsprechenden Erfahrungen aus Vietnam heimgekehrt. Auch Ketamin bewirkt - 
sowohl beim Gebrauch als Narkosemittel in der Medizin als auch bei illegalem Missbrauch als Rauschdroge - psychotische Symptome wie (vor allem visuelle) Halluzinationen, verstärkte Farbwahrnehmung sowie neuartige Körperempfindungen, Wahn, Albträume sowie Phänomene der Dissoziation: Verzerrung des Raum- und Zeitempfindens (bis zum Verlust des Zeitgefühls), das Gefühl der Verschmelzung mit der Umgebung, außerkörperliche Erfahrungen, ungewöhnliche Gedankeninhalte, Euphorie, Derealisation, Depersonalisation, Diese Erfahrungen werden meist eher positiv erlebt. Allerdings kommen auch Verwirrtheit, Delir und „Nahtoderfahrungen“ vor. Etwa ein Fünftel der Konsumenten von Ketamin berichtet über unerwünschte oder belastende Erfahrungen, etwa doppelt so viele (38\%) kennen jemand, der negative Erfahrungen gemacht hat [2]. Zu körperlichen Nebenwirkungen von Ketamin gehören Übelkeit, Erbrechen, erhöhter Speichelfluss (Hypersalivation), Blutdruckentgleisungen, Sehstörungen, Schwindel und motorische Unruhe.
Der wahrscheinlich wichtigste Wirkmechanismus von Ketamin und PCP besteht in der Blockade von postsynaptischen Ionenkanälen, den NMDA-Rezeptoren, in der Zellmembran kortikaler Neuronen. Die Substanz setzt sich dabei an eine Bindungsstelle im Inneren des lonenkanals und blockiert den Rezeptor dadurch nicht kompetitiv (kann also nicht verdrängt werden). Der physiologische Ligand dieser Rezeptoren ist der erregende Neurotransmitter Glutamat. ${ }^{3}$ NMDA-Rezeptoren leiten nicht die Erregung weiter (durch Aktivierung im Bereich von Millisekunden - dafür gibt es andere Glutamat-Rezeptoren ${ }^{4}$ ), sondern sind einige 100 Millisekunden aktiv. Sie sind im Normalzustand (wenn an der Nervenzelle ein Ruhepotenzial herrscht) durch Magnesium blockiert und werden überhaupt erst aktiv, wenn an der Synapse bereits Impulse durch Glutamat (über AMPA-Rezeptoren) übertragen werden und verstärken dann die Aktivierung. ${ }^{5}$ Im Gegensatz zu PCP wirkt Ketamin jedoch nicht nur am NMDA-Rezeptor, sondern aktiviert auch hemmende GABA-Rezeptoren von Interneuronen
[16]. Ketamin hat weiterhin eine schwache agonistische Wirkung am Opioidrezeptor. In der Psychiatrie wird Ketamin seit Jahren mit unterschiedlichem Erfolg zur Behandlung der Depression eingesetzt [3, 19, 22], wofür unterschiedlicher Wirkmechanismen vorgeschlagen wurden [26, 37]. Im peripheren vegetativen Nervensystem wirkt Ketamin hemmend auf die Wiederaufnahme von Katecholaminen wie Noradrenalin und Dopamin an der synaptischen Endplatte und verstärkt damit die Wirkungen dieser (körpereigenen oder therapeutisch gegebenen) Katecholamine (Steigerung von Blutdruck und Puls).

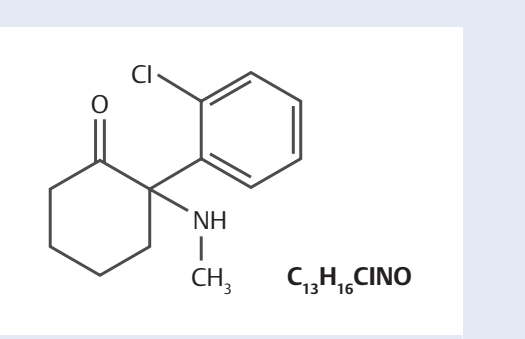

Abb. 1 Struktur- und Summenformel von Ketamin C13H16CINO nist Memantin. Auch LSD, Diazepam und Buprenorphin produzierten keine Oszillationen von 1-3 Hz. Die beiden nicht zu dissoziativen Zuständen führenden Anästhetika Dexmedetomidin und Propofol sowie eine in der Tiermedizin verwendete Mischung aus Ketamin und Xylazin führen zwar auch zu rhythmischer kortikaler Aktivität; diese war jedoch nicht auf den retrosplenialen Kortex beschränkt. „Zusammengenommen zeigen diese Daten, dass dissoziativ wirkende Substanzen, und nur diese, ein räumlich auf den retrosplenialen Kortex beschränktes oszillatorisches Muster erzeugen “, kommentieren die Autoren diese Ergebnisse [35]. Um die an diesem Geschehen beteiligten Neuronen noch genauer zu lokalisieren, exprimierten die Autoren den calmodulinassoziierten Marker nur in den Schichten 2-3 sowie in Schicht 5 der insgesamt 6 Schichten des Neokortex. Sie fanden die beschriebene rhythmische Aktivität nach der Gabe von Ketamin nur in Schicht 5 des retrosplenialen Kortex, nicht hingegen in Schicht 2-3, wo weder rhythmische Aktivität einzelner Neuronen noch synchrone rhythmische Aktivität über viele Neuronen hinweg zu finden war.

Auch elektrophysiologisch ließ sich mittels 32 Elektroden eine synchrone rhythmische Aktivität von etwa $2 \mathrm{~Hz}$ von Neuronen im retrosplenialen Kortex unter Ketamin nachweisen. Fast alle Neuronen, von denen abgeleitet wurde, feuerten synchron inner-

3 Warum heißt der NMDA-Rezeptor dann nicht einfach Glutamat-Rezeptor? - Weil diese Rezeptoren durch den Stoff N-Methyl-D-Aspartat (NMDA) aktiviert werden. Der kommt zwar im Körper nicht vor, ist aber für diesen Rezeptor-Typ hoch spezifisch und wirkt dort im Tierexperiment als Partialagonist, führt also ebenso wie der „Originalagonist“ Glutamat zur Öffnung des Ionenkanals.

4 Diese werden nach dem für sie spezifischen synthetischen Aktivator $\alpha$-Amino-3hydroxy-5-methyl-4-isoxazolpropionsäure (abgekürzt AMPA) als AMPA-Rezeptoren bezeichnet; sie sorgen für die Erregungsfortleitung und sind die verbreitetsten Neurotransmitterrezeptoren im Gehirn überhaupt.
$5 \quad$ Erst wenn die Zellmembran des postsynaptischen Neurons ausreichend vom Ruhepotenzial entfernt und depolarisiert ist, tragen auch die NMDA-Rezeptoren zum synaptischen Strom bei. Weil sie daher nur bei gleichzeitiger prä- und postsynaptischer Aktivität in Funktion treten, werden NMDA-Rezeptoren auch als molekulare Detektoren für gleichzeitige Aktivität von Neuronen - sogenannte Koinzidenzdetektoren - bezeichnet. Dass 2 mit einer Synapse verbundenen Nervenzellen gleichzeitig aktiv sind - also diejenige Nervenzelle, von der die Impulse kommen (man nennt sie im Hinblick auf diese Synapse die präsynaptische Nervenzelle) und diejenige, die diese Impulse empfängt (die postsynaptische Nervenzelle), ist im Gehirn nicht der Normalfall. Vielmehr ist das nur dann der Fall, wenn genau diese Synapse zwischen 2 Nervenzellen besonders häufig benutzt wird. Und genau dann sorgen die NMDARezeptoren dafür, dass sich die Synapse im Sinne einer Leitfähigkeitserhöhung für Aktionspotenziale ändert. Man spricht von synaptischer Plastizität und geht davon aus, dass dies der wichtigste Mechanismus für Lernen und Gedächtnis darstellt. 


\section{DER RETROSPLENIALE KORTEX}

Der retrospleniale Kortex hat dichte Verbindungen zum visuellen Kortex, anterioren Kernen des Thalamus und zur Hippocampusformation. Informationen vom Hippocampus in Form von hochfrequenten $(200 \mathrm{~Hz})$, sharp wave ripples“ werden über das Subiculum und den retrosplenialen Kortex zu Bereichen des Neokortex geleitet [27]. Beim Menschen macht er nur 0,3\% des gesamten Kortex aus, bei Nagern ist er hingegen vergleichsweise deutlich größer und nimmt bei Maus und Ratte mehr als $10 \%$ des gesamten Kortex ein ( $\triangleright$ Abb. 2 ).

Neurophysiologische Tierexperimente zur Frage, welche Informationen im retrosplenialen Kortex repräsentiert sind, zeigten seine Beteiligung bei der räumlichen Orientierung und beim episodischen Gedächtnis. Etwa 8 \% der Neuronen repräsentierten die Bewegungsrichtung des Kopfes, während andere Neuronen mit Bewegungsparametern, z. B. der Laufgeschwindigkeit korreliert waren. Zudem kodierten einige Neuronen auch Landmarken im Raum, wie schon im Jahr 1994 von der bekannten Arbeitsgruppe um Bruce McNaughton gefunden worden war. Dabei werden von den meisten Neuronen mehrere Parameter wahrscheinlich durch Projektion in Kombination repräsentiert [8]. Eine Studie an Ratten in einem langen linearen Labyrinth fand ebenfalls Neuronen, deren Aktivierung zugleich die Position im Labyrinth, die Position innerhalb der Umgebung des Labyrinths und die Drehung des Tiers nach links oder rechts repräsentierten [1].

Die beim Menschen mittels funktioneller Magnetresonanztomografie (fMRT) durchgeführten Studien bringen den retrosplenialen Kortex - bei recht guter Passung zu seiner Konnektivität und zu den tierexperimentellen Befunden - mit einer Reihe kognitiver Funktionen in Verbindung, einschließlich episodischem Gedächtnis, Navigation, Theory of Mind, Vorstellung zukünftiger Ereignisse und Verarbeitung von Szenen im Allgemeinen [34]. Diese Funktionen erscheinen zunächst sehr heterogen, sind es jedoch nicht, denn „räumliche Lokalisierung“ kann als ein Spezialfall von „Ereignis“ aufgefasst werden und steht zugleich in enger Verbindung zu Ich (meine Erfahrung) vs. Nicht-Ich (die Gedanken anderer verstehen, sich einfühlen können; also dem, was unter „theory of mind“ verstanden wird). So wundert es nicht, dass der retrospleniale Kortex bei Gedächtniskünstlern, die seit Cicero Ereignisse mit Landmarken verbinden, in entsprechenden Aufgaben vergleichsweise stärker aktiviert war [21]. Die genannten Bereiche des Gehirns überlappen mit dem sogenannten Ruhe-Netzwerk (default mode network).
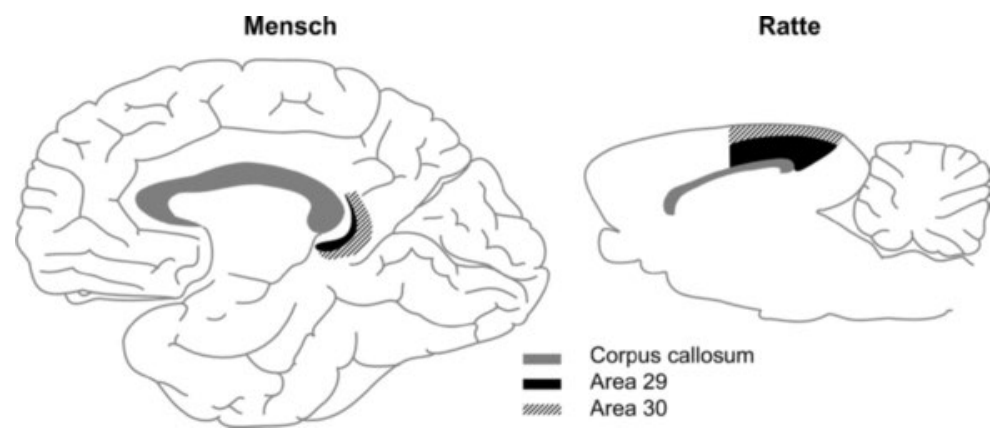

- Abb. 2 Der retrospleniale Kortex (Brodman-Area 29 und 30) bei Mensch und Ratte (nach Daten aus [23])

halb von 250 ms 5- bis 10-mal und waren dann für 250 ms stumm. Mittels gleichzeitiger Ableitung von mehreren 100 Neuronen im gesamten Gehirn wurde der $1-3 \mathrm{~Hz}$
Rhythmus auch in subkortikalen Bereichen des Gehirns nachgewiesen, die durch Kalzium-Imaging der Gehirnoberfläche nicht sichtbar gemacht werden können. Man fand mit dieser Versuchsanordnung, dass die Korrelation der Aktivität der retrosplenialen Neuronen mit Neuronen des somatosensorischen Kortex, des Subiculum, des ventralen und anteromedialen Thalamus (mit Verbindungen zum frontalen Kortex) und des Nukleus ruber, die ohne Ketamin zu beobachten war, unter Ketamin abnahm. Mit den Worten der Autoren: „Wir beobachteten eine bemerkenswerte Entkopplung zwischen benachbarten, aber unterschiedlich verbundenen Thalamuskernen. [...] Gehirnweite elektrophysiologische Experimente ergaben daher ein global nachweisbares Aktivitäts-Dissoziationsmotiv, das durch den Rhythmus und die Verdrahtung des retrosplenialen Kortex interpretiert werden kann“6[35].

Als nächsten Schritt unternahmen die Autoren umfangreiche Verhaltensexperimente. Wie angemerkt, hat Ketamin die Eigenschaft der guten Anästhesie bei gleichzeitigem Erhalt der Schutzreflexe. Im tierexperimentellen Modell (Hot-Plate-Test) lassen sich entsprechend das reflexive Zurückziehen („Flicks“) der Pfote als Reaktion auf die Erhitzung einer als Käfigboden dienenden Metallplatte auf $55^{\circ} \mathrm{C}$ von der affektiven Reaktion des Ergreifens einer Hinterpfote mit den Vorderpfoten und anschließendem Lecken an der Hinterpfote („Licks“) unterscheiden. Ketamin (in Dosen von 0, 6, 13, 25 und $50 \mathrm{mg} / \mathrm{kg}$ ) hatte keinen Einfluss auf das reflexive Verhalten (die Flicks), ab 25 mg/kg jedoch eine signifikante Reduktion des affektiven Verhaltens (Licks) zur Folge, wie - Abb. 4 zeigt. Diese Trennbarkeit von Detektion des Stimulus und affektiver Reaktion auf den Stimulus werten die Autoren als Indiz dafür, dass hier ein dissoziativer Zustand bestehen könnte.

Weitere Indizien dafür wurden in zusätzlichen Verhaltenstests gefunden, wo sich die Mäuse ab 25 mg Ketamin pro kg Körpergewicht als „affektiv weniger auslenkbar bzw. betroffen " zeigten, jedoch noch wach und responsiv waren (die Autoren schreiben „consciousness was maintained“ und weisen darauf hin, dass dies bei Dosen von mehr als 200 mg/kg nicht mehr der Fall war: „consciousness was abolished“ [35]). Entsprechend fanden die Autoren auch mit der Calzium-Imaging-Methode die synchrone Oszillation bei einer Dosis von 25 mg/kg, 

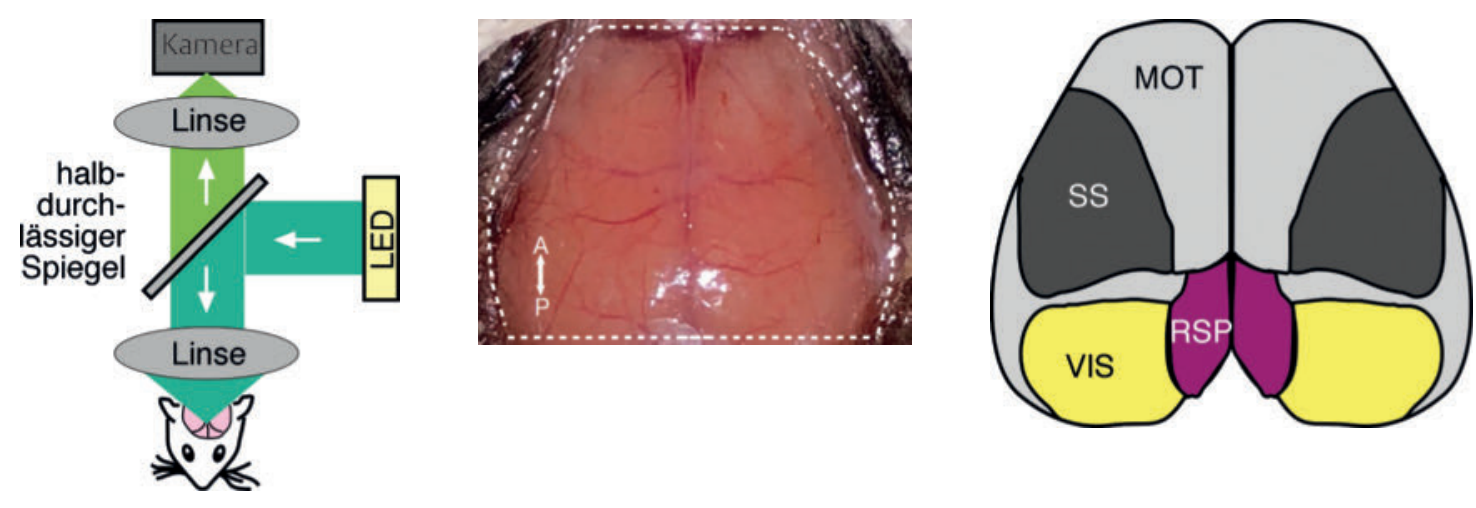

Abb. 3 Versuchsaufbau beim Calcium-Imaging (links), Sicht auf das frei präparierte Gehirn (Mitte) und schematische Darstellung der Lage des retrosplenialen Kortex (RSP) bei Mäusen (rechts; nach Daten aus [35]), MOT motorischer Kontext, SS somatosensorischer Kortex, VIS visueller Kortex, a anterior, p posterior

nicht jedoch bei einer Dosis von $13 \mathrm{mg} /$ kg. Weil Ketamin in Dosen, die Dissoziation erzeugen, auch Analgesie, Halluzinationen und Sedierung verursachen kann, wurden Verhaltenstest mit einigen der genannten psychotropen Substanzen gemacht, wobei sich zeigte, dass nur Phencyclidin (das wie Ketamin Dissoziation hervorruft) die gleichen Ergebnisse wie Ketamin in den Verhaltenstests zeigte, nicht jedoch LSD, Analgetika (Buprenorphin sowie das Lokalanästhetikum Lidocain) oder Diazepam. Mit anderen Worten: Die Verhaltensexperimente „zeigen einen messbaren und konsistenten dissoziationsartigen Phänotypen bei Mäusen, der durch erhaltene Erkennung von Reizen bei verminderter affektiver Reaktion auf sie charakterisiert ist, und der spezifisch von dissoziativ wirkenden Substanzen in Dosen, die auch das Auftreten von rhythmischer Aktivität im retrosplenialen Kortex bewirken, hervorgerufen wird "6 , halten die Autoren als Zwischenfazit fest [35]. Erst an dieser Stelle kommt die erwähnte Optogenetik ins Spiel, also der Einbau lichtempfindlicher Rezeptoren in Neurone, um sie durch Licht zu Aktionspotenzialen anzuregen ( $\boldsymbol{A} \mathbf{A b b} \mathbf{5} \mathbf{5})$. Es sollte nämlich der Nachweis geführt werden, dass es tatsächlich die unter Ketamin beobachteten Oszillation von 1-3 Hz in Schicht 5 des retrosplenialen Kortex sind, die diese Effekte der Dissoziation verursachen.

Hierzu wurde diese rhythmische Aktivität in genau diesen Neuronen (Schicht 5, retrosplenialer Kortex) dadurch erzeugt,

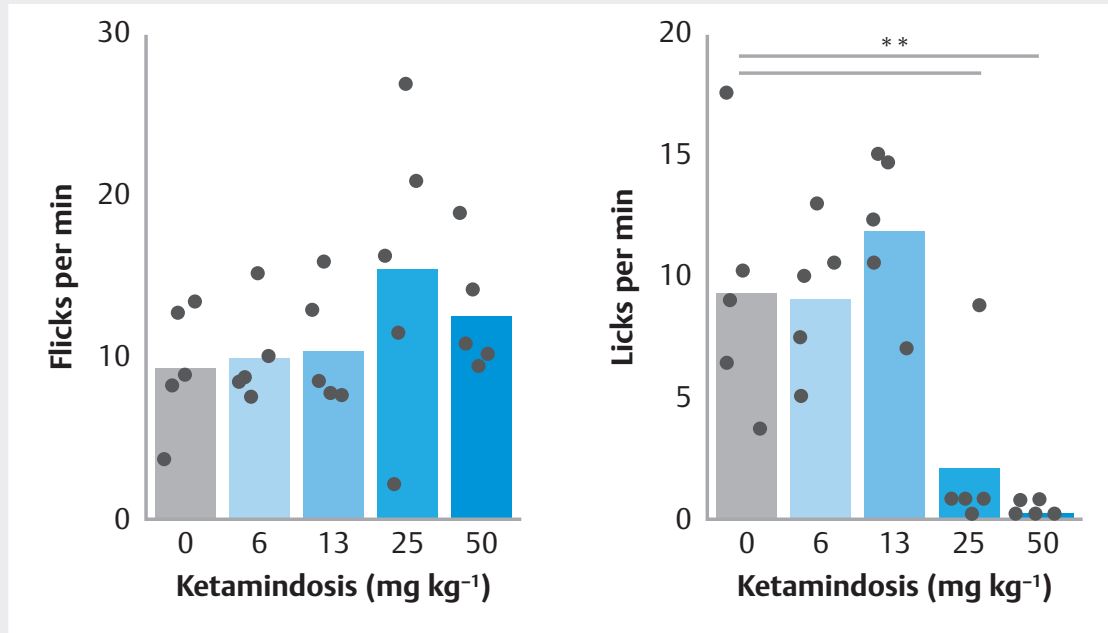

- Abb. 4 Einfluss von Ketamin in verschiedener Dosierung auf die reflexive („Flicks“) und affektive Komponente („Licks“) des Schmerzverhaltens von Mäusen. Reflexe werden nicht beeinflusst, die affektive Reaktion jedoch dosisabhängig ab 25 mg/kg Körpergewicht sehr deutlich (jeweils $n=5$; nach Daten aus [35]).

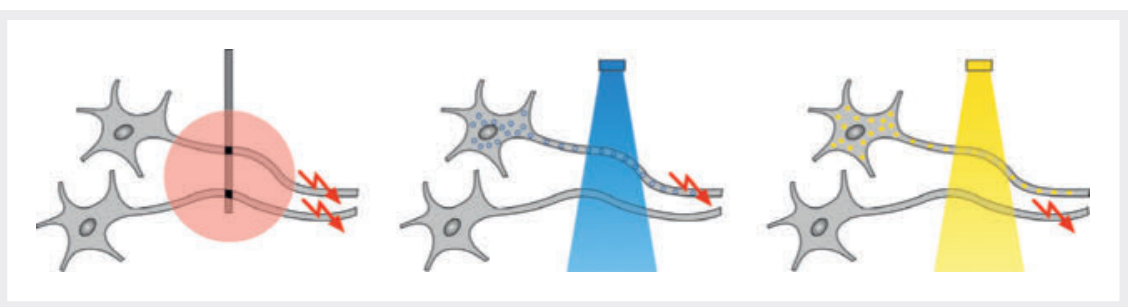

- Abb. 5 Prinzip der Optogenetik in der neurowissenschaftlichen Forschung: Gezielte Erregung (mit einem durch Blaulicht aktivierten Channelrhodopsin) oder Hemmung (mit einem durch gelbes Licht aktivierten Halorhodopsin). Im Gegensatz zur elektrischen Stimulation von Neuronen ist die Stimulation mit Licht für bestimmte Zelltypen (diejenigen, die genetisch modifiziert wurden) spezifisch und zugleich zeitlich ebenso hoch auflösend.

dass man 2 Opsine, ein blaulichtempfindliches, Aktionspotenziale produzierendes Opsin und ein auf gelbes Licht empfindliches, hemmendes (d. h. hyperpolarisieren- des) Opsin exprimierte ( $>$ Abb. 6). Durch alternierende Stimulation mit Blaulicht-Pulsen $(20 \mathrm{~Hz})$ und kontinuierlichem gelbem Licht für jeweils 250 Millisekunden konnte 


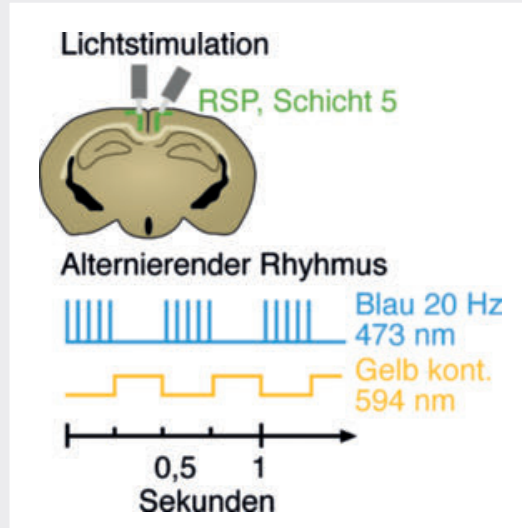

- Abb. 6 Ablauf der optogenetischen Stimulation von Neuronen im RSP, die sowohl ein erregendes als auch ein hemmendes Opsin exprimierten, um dort in Schicht 5 ohne Ketamin einen 2-Hz-Rhythmus zu erzeugen (nach Daten aus [35]).

diesen Nervenzellen ohne Ketamin ein synchroner Rhythmus von $2 \mathrm{~Hz}$,aufgedrückt“ werden. (Als Kontrollen dienten sowohl optogenetisch nicht manipulierte Mäuse als auch Mäuse mit den beiden Opsinen, die zufällig, also nicht rhythmisch stimuliert wurden.)

Zugleich führte man dann den Hot-PlateTest durch und konnte zeigen, dass die reflexhaften Reaktionen („Flicks“) nicht, die affektiven Reaktion („Licks“) jedoch - jeweils im Vergleich zu den Kontrollen - sehr wohl vermindert waren. Auch in 2 weiteren Verhaltenstests zur Messung von Dissoziation zeigte sich dieses Ergebnis. Als zusätzliche Kontrolle wurden die beiden Opsine in Zellen des somatosensorischen Kortex exprimiert, was zu keinen Unterschieden bei den Flicks und Licks führte, also zu keiner selektiven Produktion von Verhaltensweisen, die Dissoziation anzeigen. „Zusammenfassend zeigt sich auch in Abwesenheit von Ketamin bei optogenetischer Stimulation und dadurch möglicher Produktion des Aktivierungsmusters, das in Neuronen in Schicht 5 des retrosplenialen Kortex als Reaktion auf Ketamin entsteht, ein Effekt im Sinne einer Verminderung der Verbindung zwischen sensorischer und affektiver Reaktion“, kommentieren die Autoren [35].

Es trug sich zu ${ }^{6}$, dass es am Epilepsiezentrum der Stanford University einen Patienten mit Anfallsleiden gab, der über disso- ziative Auren vor den Anfällen berichtete und bei dem zum Zwecke des Monitoring der Anfälle, deren genauerer Lokalisation, Stimulation und Therapieoptimierung intrakranielle Elektroden implantiert worden waren, deren Lokalisation in der Arbeit mit „brainwide“ beschrieben wird. Das bei diesem Patienten abgeleitete intrakranielle Elektroencephalogramm (iEEG) zeigte während der Aura vor einem Anfall eine rhythmische Aktivität von 3,4 Hz - und zwar - bilateral - genau dort, wo beim Menschen das Areal liegt, das dem retrosplenialen Kortex bei der Maus entspricht (die Autoren sprechen vom posteromedialen Kortex, $\mathrm{PMC}$ ). Der nachfolgende Anfall war rechts lokalisiert.

Es zeigte sich weiterhin, dass bei $50-\mathrm{Hz}$-Stimulation dieses Bereichs rechts für etwa 1,5 Sekunden eine Aura provoziert werden konnte, bei linksseitiger Stimulation wurde über dissoziatives Erleben ohne die negativ konnotierte Erwartung eines Anfalls berichtet. Die Stimulation der Areale, in denen es zu spontanen Oszillation während der Auren kam, ergab in 11 von 13 Fällen eine dissoziative Aura, wohingegen dies praktisch an keiner anderen Stelle der Fall war. Damit wurde eine kausale Bedeutung der beteiligten Gehirnareale für die Entstehung dissoziativer Erfahrungen beim Menschen nachgewiesen. ${ }^{7}$ Es ist allerdings hervorzuheben, das die hochfrequente Stimulation dieses Gehirnareals bei nicht an Epilepsie leidenden Menschen keine Dissoziation hervorgerufen hatte, wie eine 3 Jahre zuvor ebenfalls in Stanford durchgeführte Studie mit 885 Stimulationen bei 25 Patienten gezeigt hatte [11]. Nur bei niederfrequenter synchroner Aktivierung (Maus: etwa $2 \mathrm{~Hz}$, Mensch: 3,4 Hz) kommt es damit zu dissoziativem Verhalten bzw. Erleben. Die Autoren heben in diesem Zusammenhang hervor, dass der synchron oszillierende retrospleniale Kortex mehrfach in den Thalamus projiziert: Dies führt zur funktionellen Entkopplung von sensorischen Daten vom dorsolateralen und anteriorventralen Nukleus (des Thalamus) in den posterioren Kortex von Daten, die über einen anderen thalamischen Kern (anteriormedialer Nukleus)

6 Mit den Worten der Autoren: “Together, these results demonstrated causal elicitation of human dissociative symptoms local to sites exhibiting the deep PMC rhythm" [35]. zum Frontalhirn gelangen. Dies könnte zu dissoziativen Phänomenen dadurch beitragen, dass „autobiografische Gedanken und Egozentrizität durch die Diskonnektion frontaler von posterioren kortikalen Arealen beeinträchtigt werden. Da zugleich die rhythmische Aktivität von 1-4 Hz die Kontinuität der neuronalen Aktivität in den beiden Subnetzwerken (posterior/sensorisch und anterior/cognitiv-motorisch) für Perioden von mehr als 200 ms aufrechterhält, bleibt die bewusst erlebte Geistestätigkeit erhalten - im Gegensatz zum Zustand der Anästhesie“7 [35].

Das könnte man - zumindest im Hinblick auf die sprachliche Ausgestaltung einerseits und die Entfernung zur Empirie andererseits - durchaus als Neurophilosophie bezeichnen - muss man aber nicht. Zumal die Autoren die Tragweite ihrer Befunde tatsächlich in einem größeren Zusammenhang sehen: „Die Fähigkeit [des retrosplenialen Kortex] zur unabhängigen funktionalen Verarbeitung in nur dieser kortikalen Region - wie hier aufgedeckt wurde -ist bemerkenswert im Lichte der Daten aus vielen Labors, die auf die extensiven kortiko-kortikalen Verbindungen und deren Dynamik, die den Neokortex in ein distribuiertes Netzwerk integrieren, verweisen “8 [35]. Diese nahezu Kantische Formulierung meint im Klartext ausformuliert: Wer hätte gedacht, dass Dissoziation sich an der rhythmischen Aktivität eines klitzekleinen Stückchens Kortex festmachen lässt, wo doch das allgemeine Verständnis dahin zu gehen scheint, dass „Bewusstsein“ als irgendwie „emergente Systemeigenschaft“ der gesamten Gehirnrinde zu verstehen ist. Und das ist letztlich auch die Kernaussage dieser Arbeit, mit der sich jeder auseinandersetzen muss, der über bewusstes Erleben - ich vermeide bewusst „Bewusstsein“, weil dieses Wort zu vieldeutig verwendet wird - nachdenkt.

Auch unser klinisches Denken über Dissoziation wird durch die vorliegende Arbeit grundlegend in Frage gestellt. Denn die

7 Im Original: “[...] the capability for independent functional operation of this cortical region-as revealed here-was notable in light of data from many laboratories that point to extensive cortico-cortical wiring and dynamics that integrate the neocortex into a distributed network." 
häufige Subsumierung von dissoziativen Zuständen unter „Konversionsstörungen“ ist vor dem Hintergrund der hier vorgestellten Daten problematisch: Denn „konvertiert“ wird, nach Freud, die „psychische Energie" von Affekten durch den Prozess der „Verschiebung“ auf Organe, wobei es sich um einen „Abwehrmechanismus“ handelt, der „Konflikte“ vom „Ich-Bewusstsein“ fernhalten und „das Ich“ damit schützen soll. Weil dadurch körperliches Leid - als „Affektäquivalent“ bezeichnet - entsteht, sei diese „Schutzfunktion“ pathogen. Denn die konflikthaften Gefühle (Angst) seien dem Patienten nicht mehr bewusst, sondern nur noch die Empfindungen körperlicher Reaktionen (Herzrasen, Muskelverspannung, Schwitzen, Schwindel) als deren Äquivalent.

Problematisch ist erstens, dass Konversionssymptome explizit nur nach Ausschluss einer körperlichen Erkrankung diagnostiziert werden. Das kennen wir in der Psych- iatrie und leben damit, ohne weiter darüber nachzudenken: Ein wesentlicher Teil der Schizophrenieforschung sucht nach mechanistischen, d.h. genetischen, molekularen, zytologischen, histologischen oder system-neurobiologischen Ursachen, obwohl die Krankheit seit Kurt Schneider [32] so definiert ist, dass man keine solchen findet. Nun benennt die Arbeit aus der Gruppe von Deisseroth für ein genuin psychisches Phänomen genau solche körperlichen Mechanismen. Und die Arbeit zeigt zweitens, dass unser bisheriges Modell dieses Zustandes möglicherwiese schlicht falsch ist. Nun kann man sagen, dass wir längst den „psychischen Apparat“ und die ganze mit ihm einhergehende Theorie auf den Schrotthaufen nachweislich falscher und hoffnungslos veralteter wissenschaftlicher Theorien geworfen haben ${ }^{8}$. Wenn wir diese Wörter verwenden, dann nähmen wir sie nur in metaphorischen Gebrauch, so etwa wie man ja auch bei einer Fußballmannschaft vom Mannschaftsgeist spricht, aber niemandem einfiele zu fragen, ob dieser als Stürmer oder in der Abwehr spielt. Die Theorie sei nichts anderes als ein Hilfsmittel zur Verständigung, oder wie Alfred Lorenzer [20] es formulierte, als „Tiefenhermeneutik“, die auf „szenischem Verstehen“ beruht. Dieser heute weithin üblichen Auffassung ist zu entgegnen, dass jegliches Verstehen das in den Kontext-Setzen impliziert, also Interpretamente verwendet, die außerhalb des zu Verstehenden liegen. Wie daher „szenisches“ und „ganz normales “Verstehen zu unterscheiden sein sollen, ist vollkommen unklar. Worin die „Tiefe“ der Tiefenhermeneutik besteht, lässt sich ebenso wenig angeben. Es bleibt Hermeneutik, die Lehre vom Verstehen. Eine falsche Theorie gehört nicht dazu.

Aber selbst wenn man das Weitwinkelobjektiv beim Betrachten der zusammenfassend referierten Studie wieder weglegt, kann man ganz praktisch fragen, ob die beschriebenen Phänomene und Mechanis- 
men auch für die Erfahrungen der Dissoziation bei posttraumatischer Belastungsstörung (PTBS) oder Borderline-Persönlichkeitsstörung gelten, und was das klinisch oder gar therapeutisch bedeuten könnte. Man kann vermuten, dass uns bei diesen Fragen vor allem methodische Fortschritte in der Human-Neurowissenschaft weiterbringen werden. Eines ist jetzt schon klar: Mit einer einzigen Nature-Arbeit wurde unser Denken über ein klinisch bedeutsames Phänomen der Psychopathologie vom vorletzten ins 21. Jahrhundert katapultiert.

\section{Interessenkonflikt}

Der Autor gibt an, dass kein Interessenkonflikt besteht.

\section{Korrespondenzadresse}

Prof. Dr. Dr. Manfred Spitzer

Universität Ulm

Abteilung für Psychiatrie

Leimgrubenweg 12-14

89075 Ulm

Deutschland

Literatur

[1] Alexander AS, Nitz DA. Retrosplenial cortex maps the conjunction of internal and external spaces. Nature Neuroscience 2015; 18: 1143-1151

[2] ACMD - Advisory Council on the Misuse of Drugs, Großbritannien. Ketamine: a review of use and harm. Bericht für den Innenminister und den Gesundheitsminister, London, 10. Dezember 2013 https://www.gov.uk/ government/uploads/system/uploads/attachment_data/file/264677/ACMD_ketamine_report_dec13.pdf, (abgerufen am 18.10.2020

[3] Bahji A, Vazquez GH, Zarate CA Jr. Comparative efficacy of racemic ketamine and esketamine for depression: A systematic review and meta-analysis. J Affect Disord 2020; 278: 542-555

[4] Bernstein EM, Putnam FW. Development, reliability, and validity of a dissociation scale. J Nerv Ment Dis 1986; 174: 727-735

[5] Beyeler A. Do antidepressants restore lost synapses? Ketamine triggers changes in behavior, neuron microcircuitry, and dendritic spines. Science 2019; 364: 129-130

[6] Boly M, Massimini M, Tsuchiya N, et al. Are the Neural Correlates of Consciousness in the Front or in the Back of the Cerebral Cortex? Clinical and Neuroimaging Evidence. J Neurosci 2017; 37: 9603-9613

[7] Chang H, Esteves IM, Neumann AR, et al. Coordinated activities of retrosplenial en- sembles during resting-state encode spatial landmarks. Philos Trans R Soc Lond B Biol Sci 2020; 375(1799): 20190228

[8] Chen T-W, Wardill T], Sun Y, et al. Ultra-sensitive fluorescent proteins for imaging neuronal activity. Nature 2013; 499: 295-300

[9] Chen LL, Lin L-H, Green EJ, et al. Head-direction cells in the rat posterior cortex. Experimental Brain Research 1994; 101: 8-23

[10] Deisseroth K. Optogenetics. Nature Methods 2011; 8: 26-29

[11] Foster BL, Parvizi J. Direct cortical stimulation of human posteromedial cortex. Neurology 2017; 88: 685-691

[12] Fox KCR, Foster BL, Kucyi A, et al. Intracranial electrophysiology of the human default network, Trends Cogn Sci 2018; 22: 307-324

[13] Freyberger H], Spitzer C, Stieglitz RD,et al. Fragebogen zu dissoziativen Symptomen (FDS): Deutsche Adaptation, Reliabilität und Validität der amerikanischen Dissociative Experience Scale (DES). Psychother Psychosom med Psychol 2018; 48: 223-229

[14] Freud S. Studien über Hysterie. Frühe Studien zur Neurosenlehre. Gesammelte Werke. Frankfurt am Main: Fischer, 1895/1952

[15] Hallett M. Functional (psychogenic) movement disorders - Clinical presentations. Parkinsonism Relat Disord 2016; 22 Suppl 1: S149-52

[16] Hevers W, Hadley SH, Lüddens H, et al. Ketamine, But Not Phencyclidine, Selectively Modulates Cerebellar GABAA Receptors Containing $\alpha 6$ and $\delta$ Subunits. Journal of Neuroscience 2008; 28: 5383-5393

[17] Hung CC, Liu YH, Huang CC, et al. Effects of early ketamine exposure on cerebral gray matter volume and functional connectivity. Sci Rep 2020; 10: 15488

[18] Kovar K-A. Designer-Drogen der Phencyclidin-Reihe. Pharmazie in unserer Zeit 1994; 23

[19] Lipsitz O, McIntyre RS, Rodrigues NB, et al. Early symptomatic improvements as a predictor of response to repeated-dose intravenous ketamine: Results from the Canadian Rapid Treatment Center of Excellence. Prog Neuropsychopharmacol Biol Psychiatry 2020; 105: 110126

[20] Lorenzer A. Die Wahrheit der psychoanalytischen Erkenntnis. Ein historisch-materialistischer Entwurf. Frankfurt am Main: Suhrkamp, 1974

[21] Maguire EA, Valentine ER, Wilding JM, et al. Routes to remembering: the brains behind superior memory. Nat Neurosci 2003; 6 : 90-95

[22] McIntyre RS, Rodrigues NB, Lipsitz O, et al. The effectiveness of intravenous ketamine in adults with treatment-resistant major depressive disorder and bipolar disorder presenting with prominent anxiety: Results from the Canadian Rapid Treatment Center of Excellence. J Psychophar- macol 2020; 269881120954048. doi: 10.1177/0269881120954048

[23] Miller AMP, Mau W, Smith DM. Retrosplenial Cortical Representations of Space and Future Goal Locations Develop with Learning. Current Biology 2019; 29: 2083-2090

[24] Mitchell AS, Czajkowski R, Zhang N, etz al. Retrosplenial cortex and its role in spatial cognition. Brain and Neuroscience Advances 2018; 2: 1-13

[25] Miyasaki JM, Sa DS, Galvez-Jimenez N, et al. Psychogenic movement disorders. Can J Neurol Sci 2013; 30 Suppl 1: S94-100

[26] Moda-Sava RN, Murdock MH, Parekh PK, et al. Sustained rescue of prefrontal circuit dysfunction by antidepressant-induced spine formation. Science 2019; 364 (6436): eaat8078

[27] Nitzan N, McKenzie S, Beed P, et al. Propagation of hippocampal ripples to the neocortex by way of a subiculum-retrosplenial pathway. Nat Commun 2020; 11: 1947

[28] Priebe K, Schmahl C, Stiglmayr C. Dissoziation: Theorie und Therapie. Berlin: Springer, 2013

[29] Pschyrembel W et al. Klinisches Wörterbuch, Stichwort Dissoziation. Berlin: De Gruyter, 2017

[30] Serafini G, Howland RH, Rovedi F, et al. The role of ketamine in treatment-resistant depression: a systematic review. Curr Neuropharmacol 2014; 12: 444-461

[31] Smith K. Method man: Karl Deisseroth is leaving his mark on brain science one technique at a time. Nature 2013; 497: 550-552

[32] Schneider K. Klinische Psychopathologie. Berlin: Springer, 1959

[33] Solt K, Akeju O. An altered state of consciousness illuminated. Nature 2020; 586: 31-32

[34] Vann S, Aggleton J, Maguire E. What does the retrosplenial cortex do?. Nat Rev Neurosci 2009; 10: 792-802

[35] Vesuna S, Kauvar IV, Richman E, et al. Deep posteromedial cortical rhythm in dissociation. Nature 2020; 586: 87-94

[36] Wang C, Zheng D, Xu J, et al. Brain damages in ketamine addicts as revealed by magnetic resonance imaging. Front Neuroanat 2013; 7: 23

[37] Yang Y, Cui Y, Sang K, et al. Ketamine blocks bursting in the lateral habenula to rapidly relieve depression. Nature 2018; 554(7692): 317-322

\section{Bibliografie}

DOI https://doi.org/10.1055/a-1193-8661 Nervenheilkunde 2020; 39: 847-854 (c) 2020. Thieme. All rights reserved. Georg Thieme Verlag KG, Rüdigerstraße 14, 70469 Stuttgart, Germany ISSN 0722-1541 